\title{
HIGH FIN WIDTH MOSFET USING GAA STRUCTURE
}

\author{
S.L.Tripathi ${ }^{1}$, Ramanuj Mishra ${ }^{2}$, R.A.Mishra ${ }^{3}$ \\ Department of Electronics and Communication Engineering, MNNIT, Allahabad
}

\begin{abstract}
This paper describes the design and optimization of gate-all-around (GAA) MOSFETs structures. The optimum value of Fin width and Fin height are investigated for superior subthreshold behavior. Also the performance of Fin shaped GAA with gate oxide HfO2 are simulated and compared with conventional gate oxide SiO2 for the same structure. As a result, it was observed that the GAA with high $K$ dielectric gate oxide has more possibility to optimize the Fin width with improved performance. All the simulations are performed on 3-D TCAD device simulator.
\end{abstract}

\section{KEYWORDS}

Gate all around(GAA),TG FinFET, High K gate oxide, Silicon-On-Insulator(SOI), Work function, Short channel effect, DIBL, Subthreshold Slope,3-D Sentaurus TCAD tool.

\section{INTRODUCTION}

Non planar three-dimensional devices with multiple gates [1] are more promising candidate for high current drive capability and better short-channel characteristics. The use of ultra-thin body (UTB) and Multiple Gate SOI structures allows the fabrication of fully-depleted devices that offer not only extremely good control of SCEs but also a very good behavior with respect to drain induced barrier-height lowering (DIBL), threshold voltage roll-off, and off-state leakage [1]. DG FinFET[2] is one of the example of non planner multigate MOSFET with superior performance than planner DG MOSFET. The Fin width $\left(\mathrm{W}_{\text {fin }}\right)$ and height $h_{\text {fin }}$ characterises the FinFET(Fig1) structure. The channel width $\left(\mathrm{W}=\mathrm{W}_{\text {fin }}+2 h_{\text {fin }}\right)$ of FinFET [3] can be increased without increasing the actual layout area, simultaneously improving the on state current. The current drive of multiple-gate SOI MOSFETs [4-5] is essentially proportional to the total gate width. Considering a pitch $\mathrm{P}$ for the fins, the current in a multigate device[1] is given by:

$$
\mathrm{I}_{\mathrm{D}}=\mathrm{I}_{\mathrm{D}_{0}} \frac{\theta \mu_{\text {top }} \mathrm{W}_{\text {fin }}+2 \mu_{\text {side }} \mathrm{h}_{\text {fin }}}{\mu_{\text {top }} P}
$$

Where,

$\theta$

$\mathrm{I}_{\mathrm{D} 0}$ : Current in single gate

DOI : $10.5121 /$ vlsic.2012.3509 
International Journal of VLSI design \& Communication Systems (VLSICS) Vol.3, No.5, October 2012

$\mathrm{W}_{\text {fin }}$ : Width of each individual Fin

$\mathrm{h}_{\mathrm{fin}}$ : Height of each individual Fin

$\boldsymbol{\theta}: 1$, for Triple gate FinFET

Additionally, lots of efforts are being made to enhance the FinFET structure. The important aspects of different FinFET structures are better subthreshold swing (SS) and drain-induced barrier lowing (DIBL) which is possible with optimum ratio of gate length to Fin width[6-7]. It has been widely known that the Fin width in DG MOSFETs should be less than 0.7 times the gate length for proper suppression of short-channel effects [8]. Therefore, the dimension in devices is determined by the fin width mainly and not by the gate length.
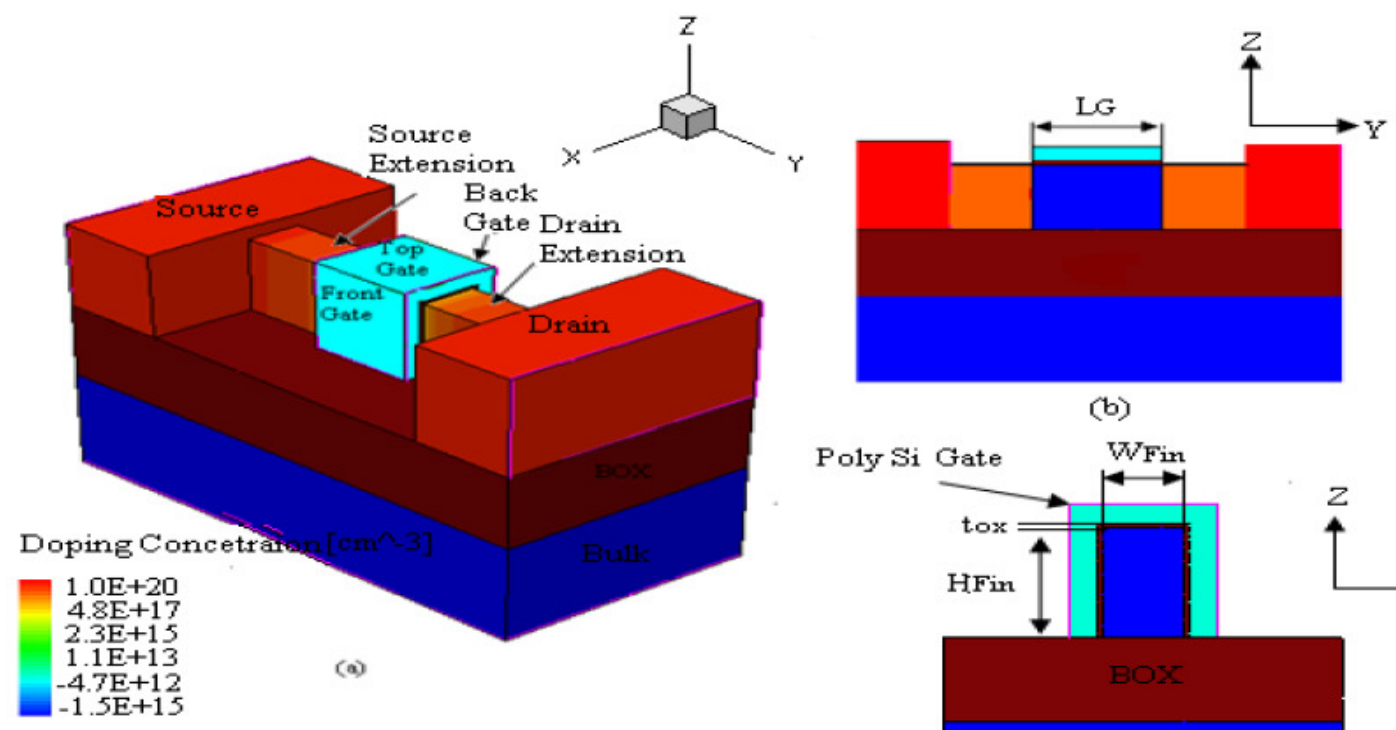

(b)

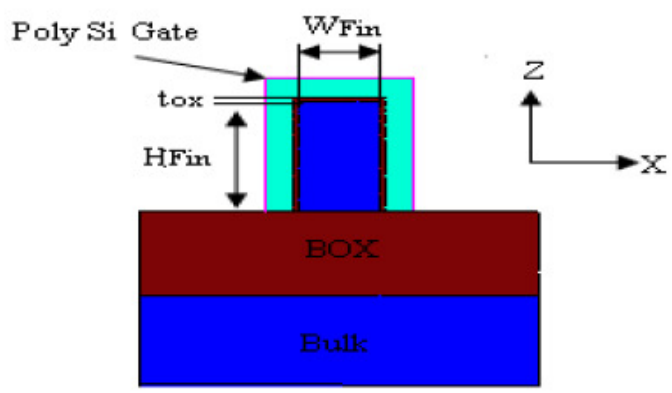

(c)

Fig. 1 (a) 3D Structure of TG FinFET (b) Front view of FinFET, (c) Fin structure near Gate

This paper deals with improvement of subthreshold performance of gate-all -around(GAA) structure over tri-gate FinFET structure. The gate-all-around (GAA) MOSFETs[9] in which the gate oxide and the gate electrodes wrap around the channel region exhibit excellent transconductance and short-channel behavior because of the presence of two additional inversion channels (at the top and the bottom of the silicon fin) and the occurrence of strong volume inversion[10]. Using GAA MOSFETs can lead to the increase of the ratio of the Fin width to the gate length. If the parameters of GAA MOSFETs are optimized, the short-channel effects are adequately suppressed even if the fin width is larger than the gate length.

Three-dimensional (3-D) simulations were performed for TG and GAA MOSFETs with various fin widths, Fin heights, and gate-oxide thicknesses to explore short-channel effects. Further, the GAA structure is simulated with high K-dielectric material (HfO2) and it shows better characteristic performance. Further, conventional GAA structure using $\mathrm{SiO} 2$ as dielectric material, is compared with GAA structure using high K-dielectric material (HfO2). 


\section{DEVICE STRUCTURE}

Fig. 2 shows the bird's eye views GAA MOSFETs used in device simulations. Device simulations were performed by the 3-D TCAD simulator [11-12]. SOI substrate was used in both TG FinFET and GAA MOSFETs having n-type channels and the same physical parameters as follows. Basically, the tri-gate FinFET designed is of $32 \mathrm{~nm}$ channel length with source/drain doping is $1 \mathrm{E} 20 \mathrm{~cm}^{-3}$ (n type). Metal is used as gate contact material and the work function of metal is kept $4.62 \mathrm{eV}$. Silicon dioxide is as gate oxide material. Gate oxide thickness is kept $1.1 \mathrm{~nm}$. The channel doping is 1E16(p type) with $\mathrm{V}_{\mathrm{DD}}=1.0 \mathrm{~V}$. The FinFET is designed with, 10nm Fin width and $60 \mathrm{~nm}$ Fin height while GAA is designed with different Fin width between 10nm to $30 \mathrm{~nm}$ and Fin height between $20 \mathrm{~nm}$ to $60 \mathrm{~nm}$. For first section of results the gate oxide is material used $\mathrm{SiO} 2$ and secondly it is designed with $\mathrm{HfO} 2$.

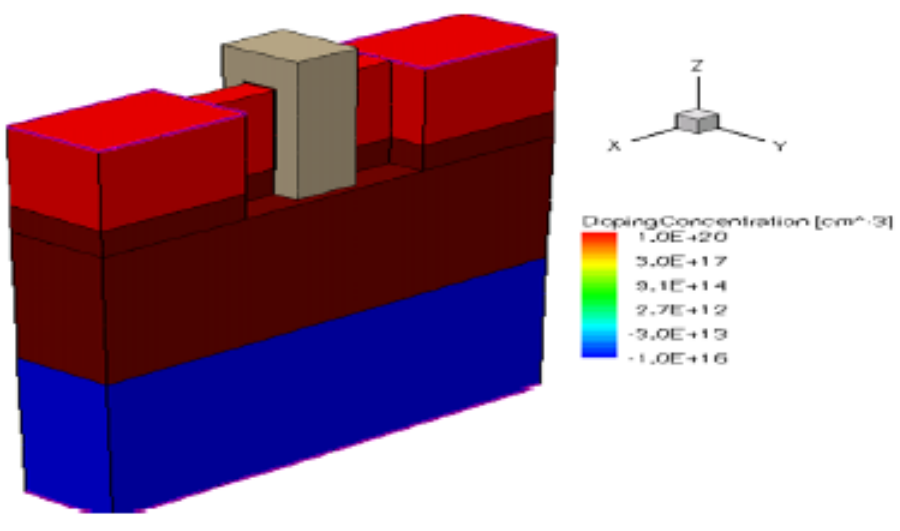

(a)

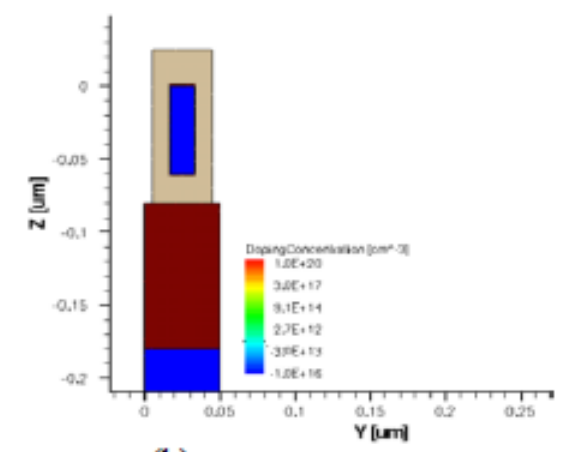

(b)

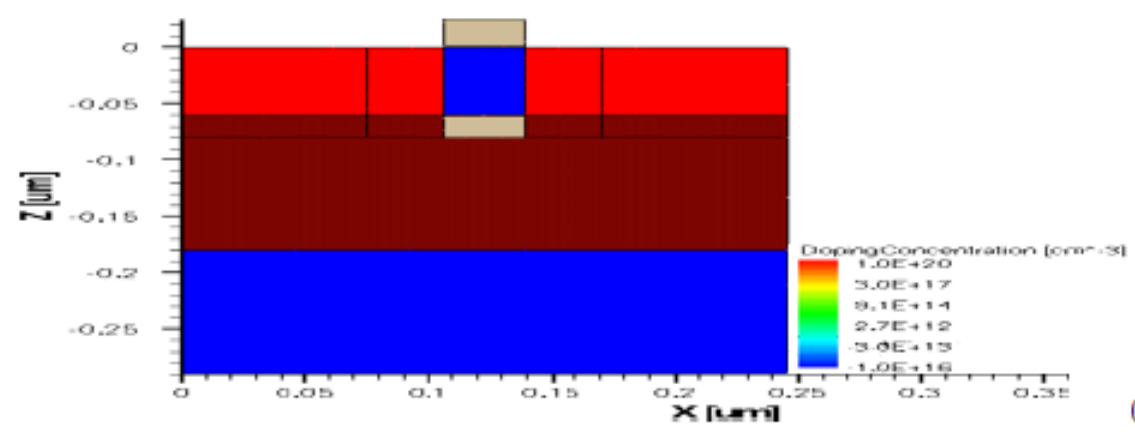

(c)

Fig. 2 (a) 3-d structure of GAA MOSFET (b) Y-cut of 3-d GAA structure (c) X-cut of 3-d GAA structure

\section{SIMULATION AND RESULTS}

For the conventional Bulk FinFET with low channel doping (1E16) Subthreshold slope of 91.78 $\mathrm{mV} /$ decade while for SOI FinFET Subthreshold slope of $68.57 \mathrm{mV} /$ decade. Therefore we can 
International Journal of VLSI design \& Communication Systems (VLSICS) Vol.3, No.5, October 2012

improve subthreshold performance with the use of SOI technology. We can also improve the performance with the increase in channel doping (1E18) for which the subthreshold slope is 79.18 $\mathrm{mV} /$ decade. But for low channel doping SOI FinFET is preferred because of its better on current drive $\left(\mathrm{I}_{\mathrm{ON}}\right)$ and lower delay characteristics. Further we can improve the ON/OFF characteristics with the optimum design of GAA structure.

\subsection{Effects of $\mathbf{W}_{\text {fin }}$ and $\mathbf{h}_{\text {fin }}$ variation on GAA structure}

$\mathrm{V}_{\text {th }}$ plays important role in scaling of device with different technology. In GAA MOSFET structure, $\mathrm{V}_{\text {th }}$ is the function of $\mathrm{W}_{\text {fin }}$ and $\mathrm{h}_{\text {fin }}$. Change in these parameters shows that for different value of it we cannot use same work function and channel doping. Therefore the need of $V_{\text {th }}$ adjustment is required for each $\mathrm{h}_{\text {fin }}$ and $\mathrm{W}_{\text {fin }}$ variations. Effect of $\mathrm{W}_{\text {fin }}$ on $\mathrm{V}_{\text {th }}$ variation and sensitivity of $\mathrm{I}_{\text {off }}$ (i.e.effect of $\mathrm{W}_{\text {fin }}$ variation on $\mathrm{I}_{\text {off }}$ ) is more with high value of $\mathrm{h}_{\text {fin }}$ as shown in Fig. 3.1, 3.2and 3.3. So, if we optimize the device for high value of $\mathrm{W}_{\text {fin }}$ then the lower value of $\mathrm{h}_{\text {fin }}$ is needed. But we require high value of $h_{\text {fin }}$ to get higher on current due to high value of gate length. Also high $\mathrm{h}_{\text {fin }}$ leads to better area efficiency. Therefore, we need to optimize the $\mathrm{W}_{\text {fin }}$ and $\mathrm{h}_{\mathrm{fin}}$ to get superior performance.

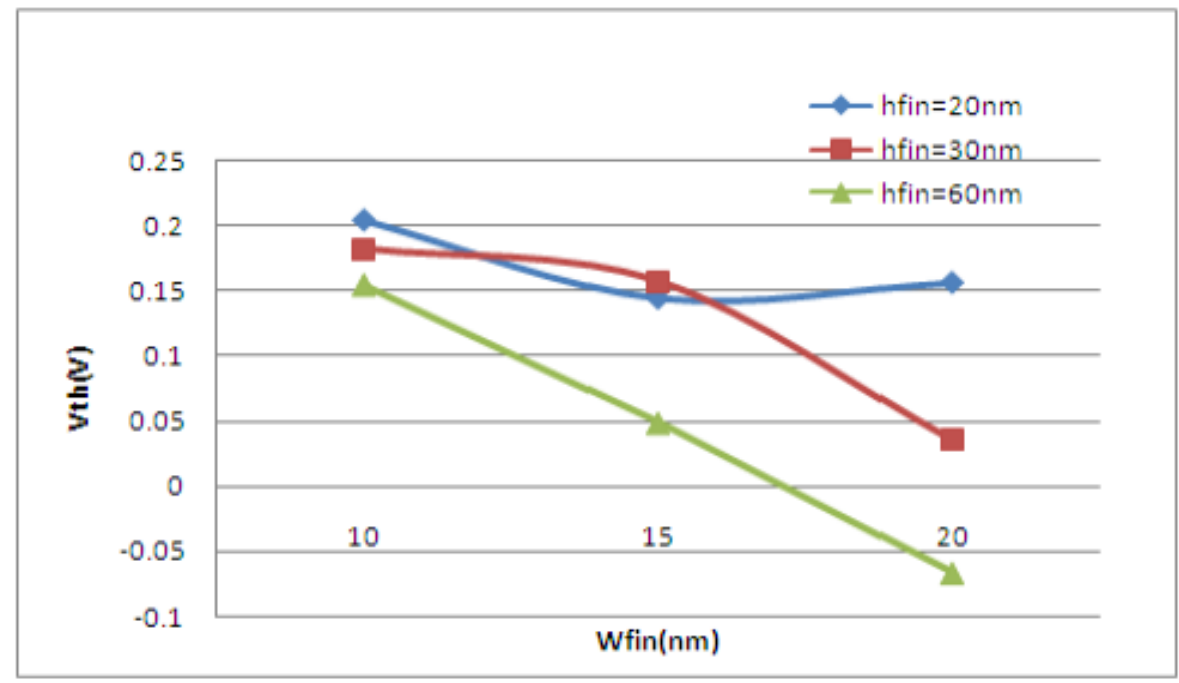

Fig. $3.1 \mathrm{~V}_{\text {th }}$ variation with $\mathrm{W}_{\text {fin }}$ for different $\mathrm{h}_{\text {fin }}$ 
International Journal of VLSI design \& Communication Systems (VLSICS) Vol.3, No.5, October 2012

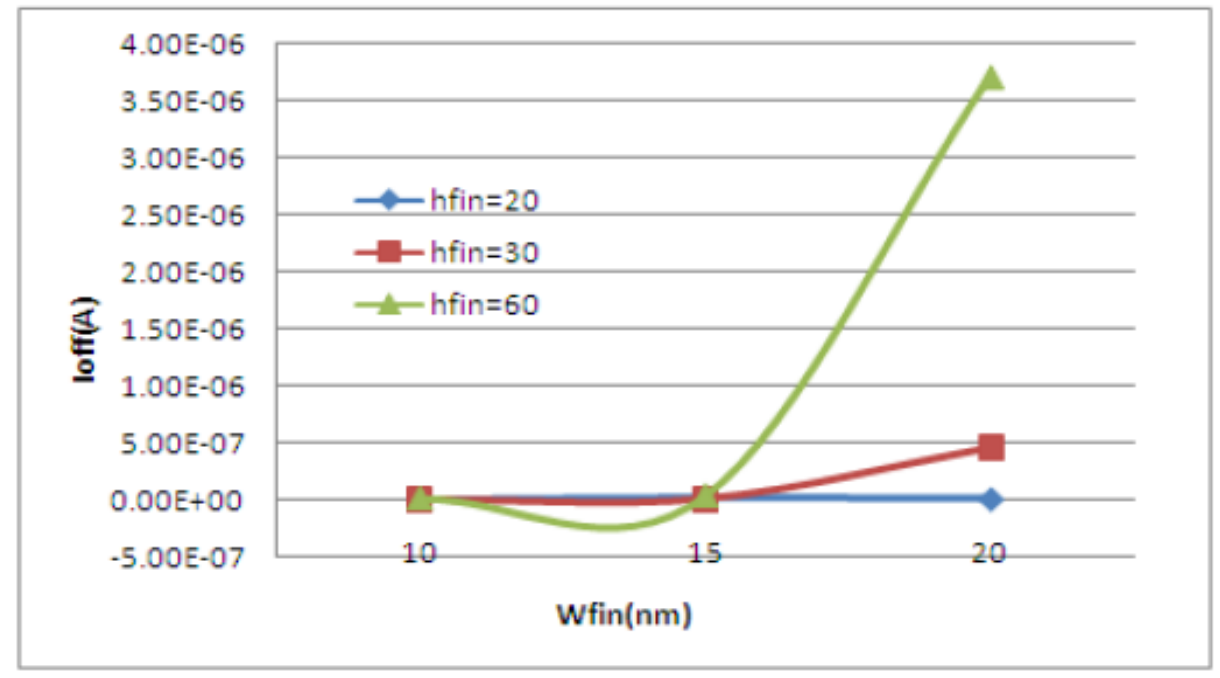

Fig. 3.2 $\mathrm{I}_{\text {off }} \mathrm{Vs} \mathrm{W}_{\text {fin }}$ for different $\mathrm{h}_{\text {fin }}$

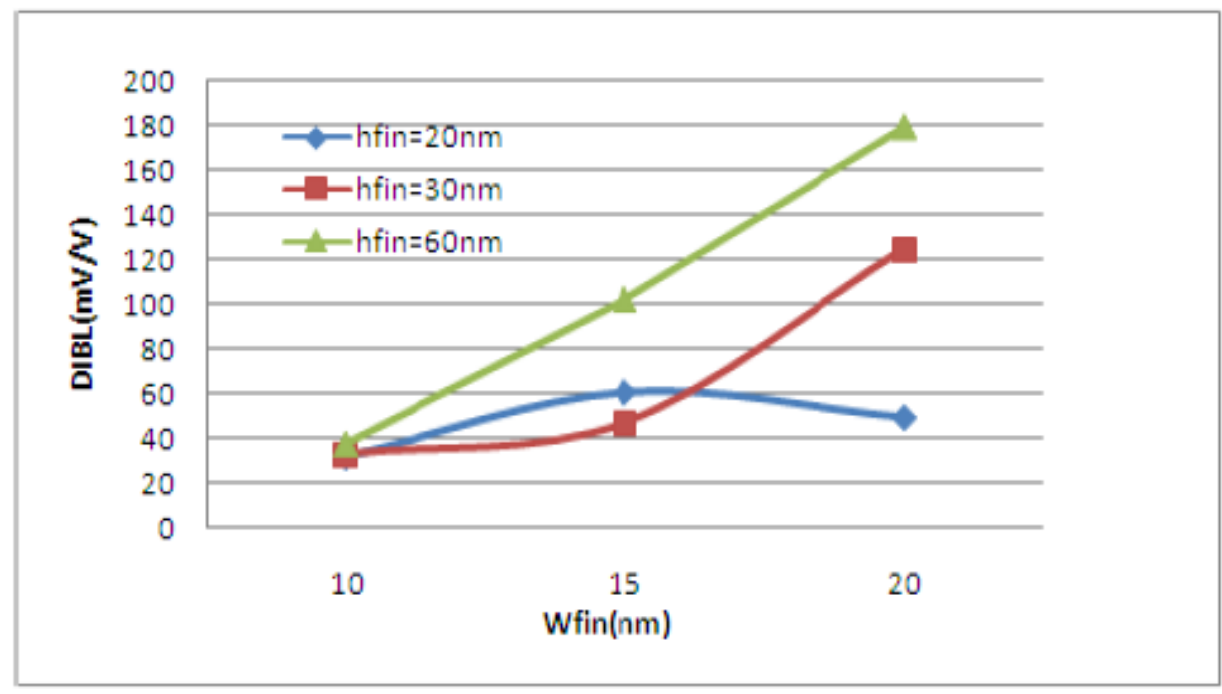

Fig. 3.3 DIBL Vs $\mathrm{W}_{\text {fin }}$ of GAA structure

Fig. 3.4, 3.5, 3.6 and 3.7 shows the different performance parameter of GAA structure with changing value of $\mathrm{h}_{\text {fin }}$. The increase or decrease in performance parameters with increasing value of $\mathrm{h}_{\text {fin }}$, is also dependent on $\mathrm{W}_{\text {fin }}$. Like for $\mathrm{W}_{\text {fin }}=10 \mathrm{~nm}$ we got best results at high value of $\mathrm{h}_{\mathrm{fin}}=60 \mathrm{~nm}$. For $\mathrm{W}_{\text {fin }}=15 \mathrm{~nm}$ the best results are obtained at $\mathrm{h}_{\mathrm{fin}}=30 \mathrm{~nm}$. But if we further decrease $\mathrm{h}_{\text {fin }}$ upto 20nm performance degrades. Similarly, for $\mathrm{h}_{\text {fin }}=20 \mathrm{~nm}$ the performance degrades at $\mathrm{W}_{\text {fin }}=20 \mathrm{~nm}$ and the best results are obtained at $\mathrm{h}_{\text {fin }}=30 \mathrm{~nm}$ which is higher than optimized Fin height $\left(\mathrm{h}_{\text {fin }}\right)$ of $\mathrm{W}_{\text {fin }}=15 \mathrm{~nm}$. 
International Journal of VLSI design \& Communication Systems (VLSICS) Vol.3, No.5, October 2012

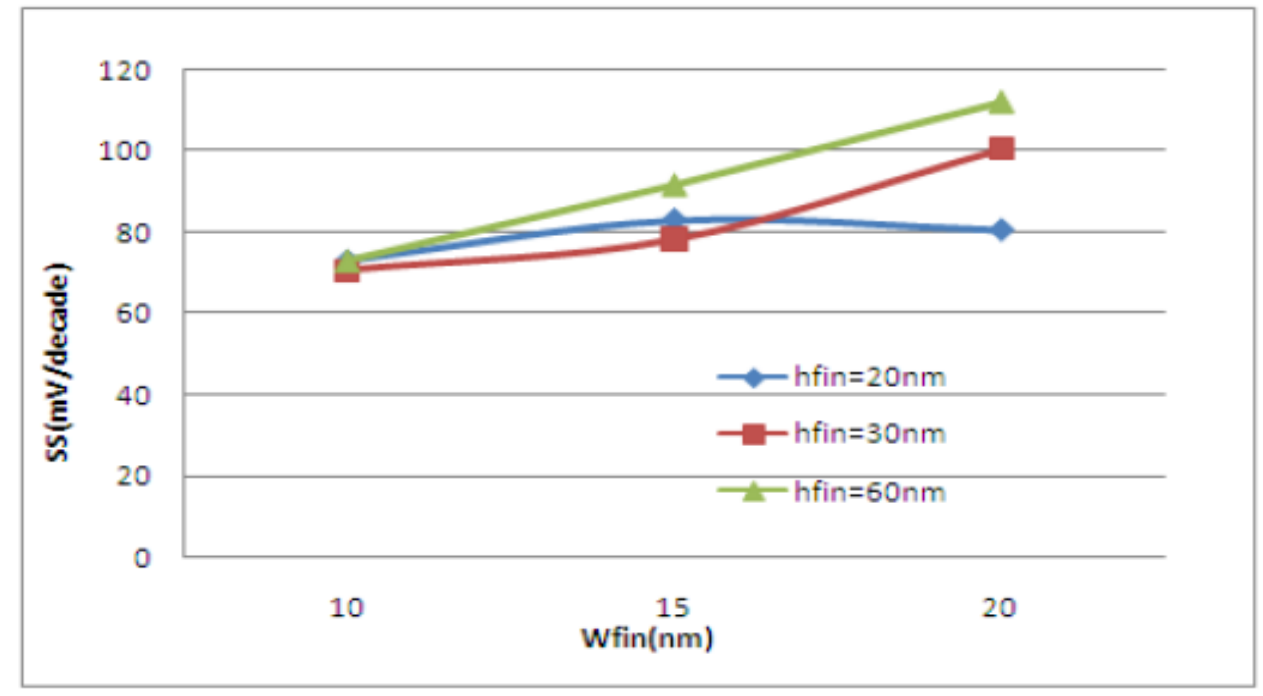

Fig. 3.4 SS Vs $\mathrm{W}_{\text {fin }}$ of GAA structure

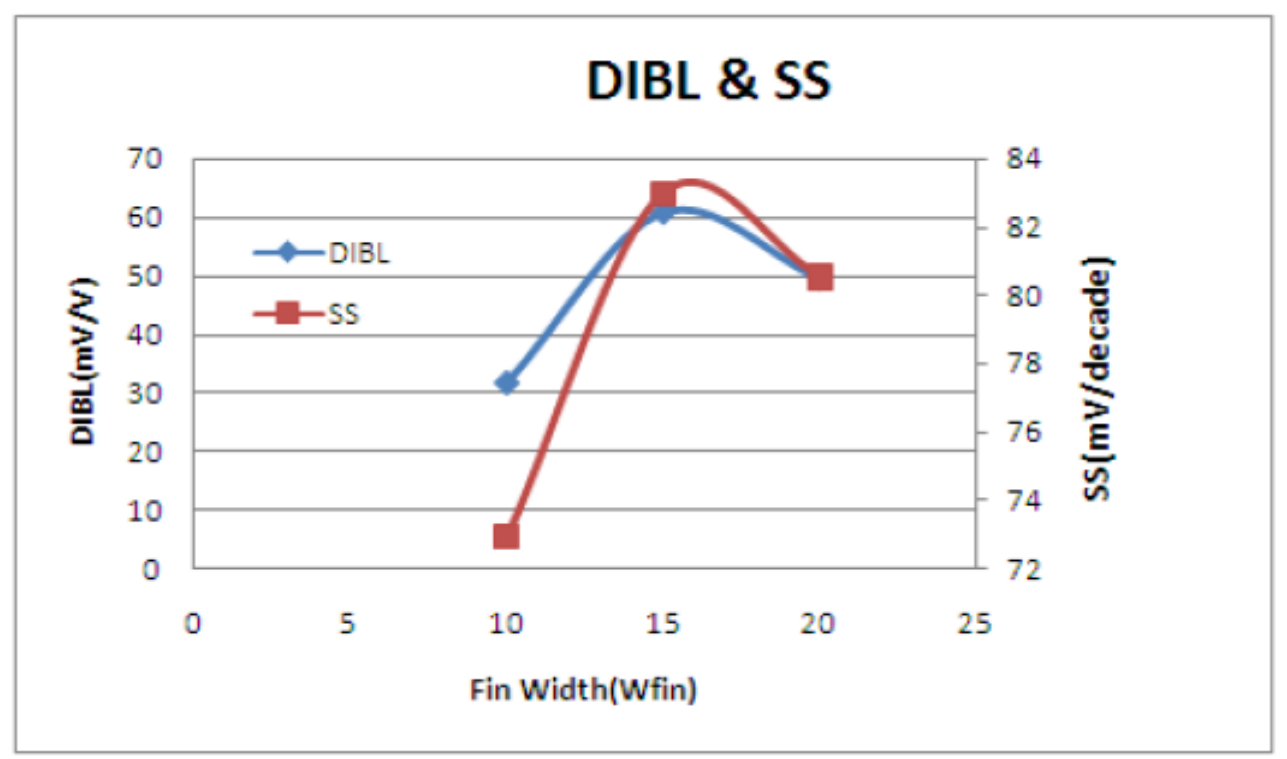

Fig. 3.5 DIBL and SS for different $\mathrm{W}_{\text {fin }}$

Fig 3.6 indicate that Fin width increment degrades subthreshold performance if Fin height is taken $30 \mathrm{~nm}$ but in next Figure we have observe a significant advantage of having high fin width device its high value of Ion for $\mathrm{VDD}=1.5 \mathrm{~V}$, almost twice current is obtained for $\mathrm{W}_{\text {fin }}=20$ as compare to $\mathrm{W}_{\text {fin }} 10 \mathrm{~nm}$ and $15 \mathrm{~nm}$. Even for $\mathrm{VDD}=1 \mathrm{~V}$, the amount of Ion is significantly large. So the use of high fin width might solve the problem of low on current value associated with nanoelectronics devices. 


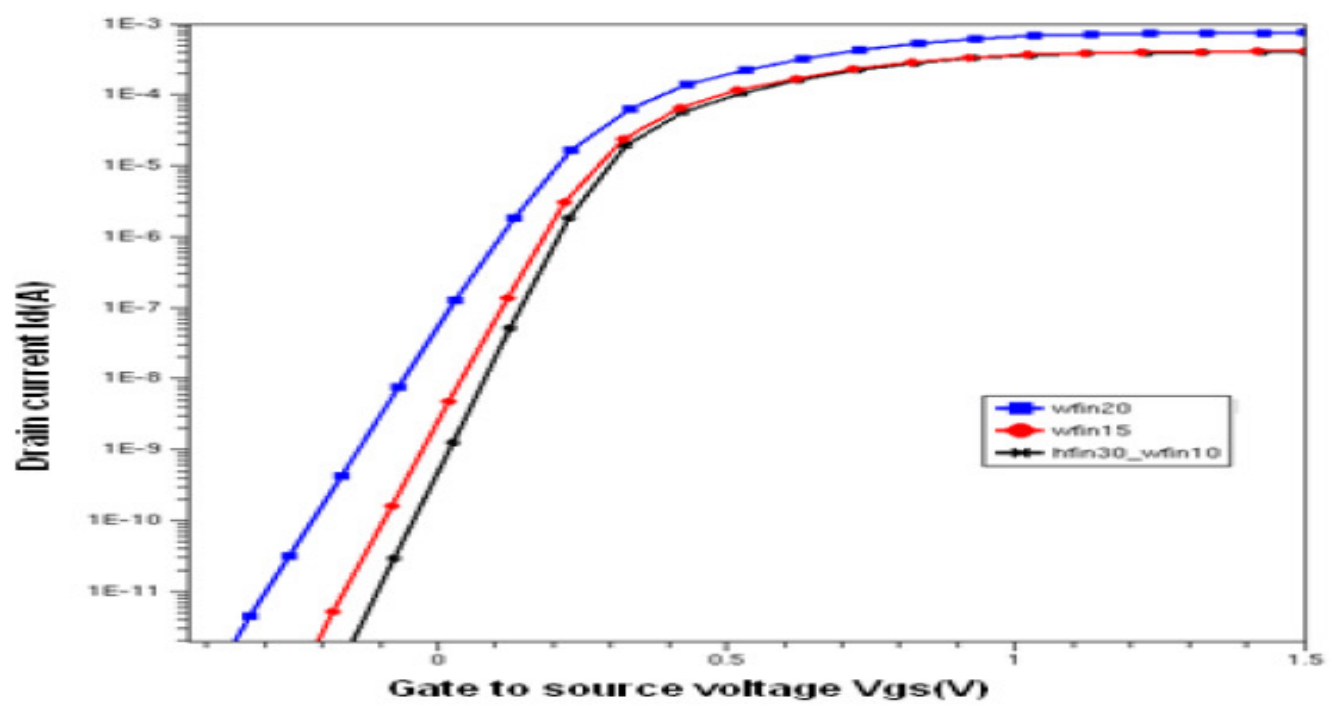

Fig. 3.6 Subthreshold characteristics of GAA structure for different fin width

From Fig. 3.7 ION Vs $\mathrm{W}_{\text {fin }}$ using GAA structure of $\mathrm{h}_{\text {fin }}=60 \mathrm{~nm} \& \mathrm{~W}_{\text {fin }}=10 \mathrm{~nm}, \mathrm{~h}_{\text {fin }} 30 \mathrm{~nm} \&$ $\mathrm{W}_{\text {fin }}=20 \mathrm{~nm}$, the same range of Ion is obtained. So, use of higher fin width can be useful for maintaining proper driving current (on current) while using shallow junction ( low $\mathrm{h}_{\text {fin }}$ ) devices. Therefore, we can make junction more shallower ( lower $\mathrm{h}_{\text {fin }}$ ) for improvement in short channel performance. From Fig. 3.5, 3.7 of DIBL, SS and Ion, it is observed that the performance of $" \mathrm{~h}_{\mathrm{fin}}=60 \mathrm{~nm} \quad \& \quad \mathrm{~W}_{\mathrm{fin}}=10 \mathrm{~nm}, \mathrm{~h}_{\mathrm{fin}}=30 \mathrm{~nm} \quad \& \quad \mathrm{~W}_{\text {fin }}=15 \mathrm{~nm}, \mathrm{~h}_{\text {fin }}=20 \mathrm{~nm} \quad \& \quad \mathrm{~W}_{\text {fin }}=20 "$ GAA are comparable. In, Fig.3.5 DIBL and SS is shown for $\mathrm{h}_{\mathrm{fin}}=20 \mathrm{~nm}$ and variable $\mathrm{W}_{\text {fin }}$ which indicates that performance does not always degrades with increase in $\mathrm{W}_{\text {fin. }}$. For further improvement in high fin width devices (if high $\mathrm{W}_{\text {fin }}$ is used) the proper matching of short channel characteristics are needed to maintain performances in desirable range. So, here we discuss the scope of high fin width GAA using high $\mathrm{K}$ gate materials.

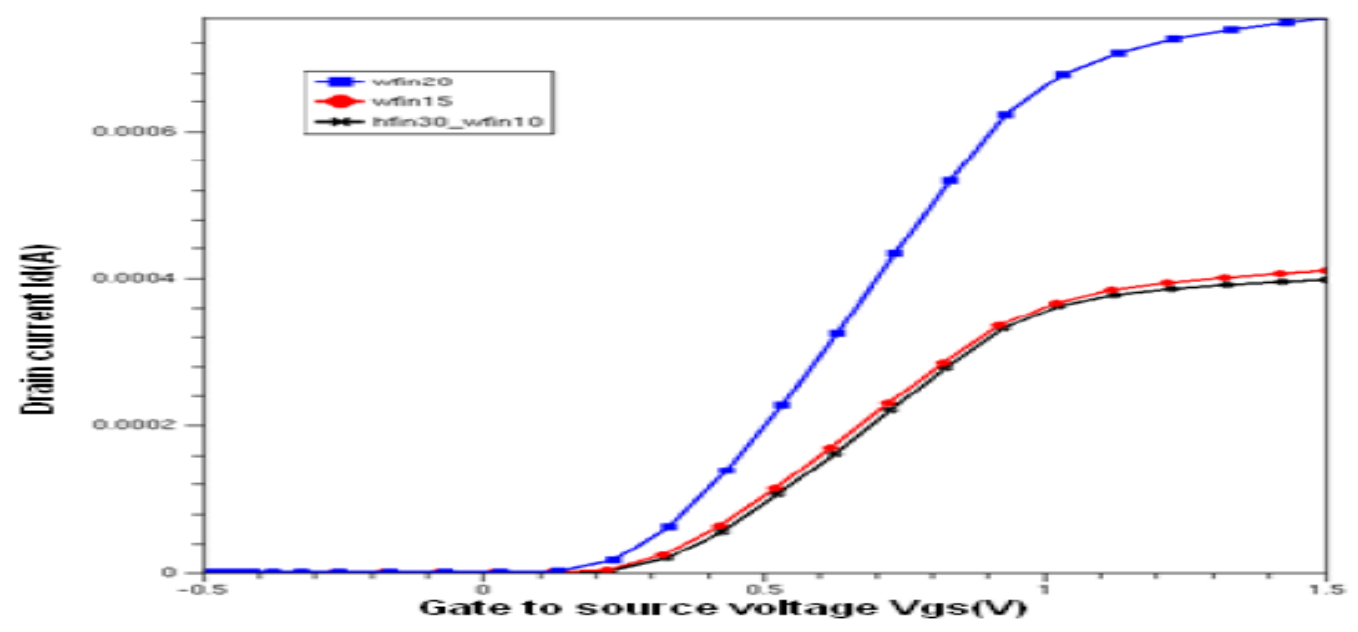

Fig.3.7 Transfer characteristics of GAA structure for different $\mathrm{W}_{\text {fin }}$ 
International Journal of VLSI design \& Communication Systems (VLSICS) Vol.3, No.5, October 2012

\subsection{Effect of high K dielectric material on GAA structure}

The use of high $\mathrm{K}$ materials [13-14] as gate oxide results in to the increase in on state current while off state current, subthreshold slope and DIBL decreases, enhancing the FinFET performance due to the fringing electric field[15]. The magnitude of the fringing electric field depends upon the dielectric constant of the medium in which it is getting leaked. The fringing field increases with the use of high $\mathrm{k}$ dielectric material for gate oxide. The different high $\mathrm{K}$ dielectric material such as Silicon Oxide (SiO2), Silicon Nitride (Si3N4) and Hafnium Oxide (HfO2) having dielectric constants of 3.5, 7.5 and 25 respectively, can be utilized at different places in different MOS devices for performance improvement.

Similarly, in case of GAA structure high K material(HfO2) gate oxide can be used to get improved subthreshold performance with high $\mathrm{W}_{\text {fin }}$. It is clear from the characteristics of Fig.3.8 that there is an increase in on state current of the device with the use of high $\mathrm{K}$ dielectric material. Simultaneously, the off-state leakage current of the GAA structure decreases with the use of high $\mathrm{K}$ dielectric materials as in Fig.3.9. The decrease in off state current is basically because of increase in the barrier potential faced by the carriers in case of high $\mathrm{K}$ dielectric materials. The increase in the Ion/Ioff ratio of the device which is very essential for low power operation of the device and the value of it can be controlled by proper design of $\mathrm{W}_{\text {fin }}$.

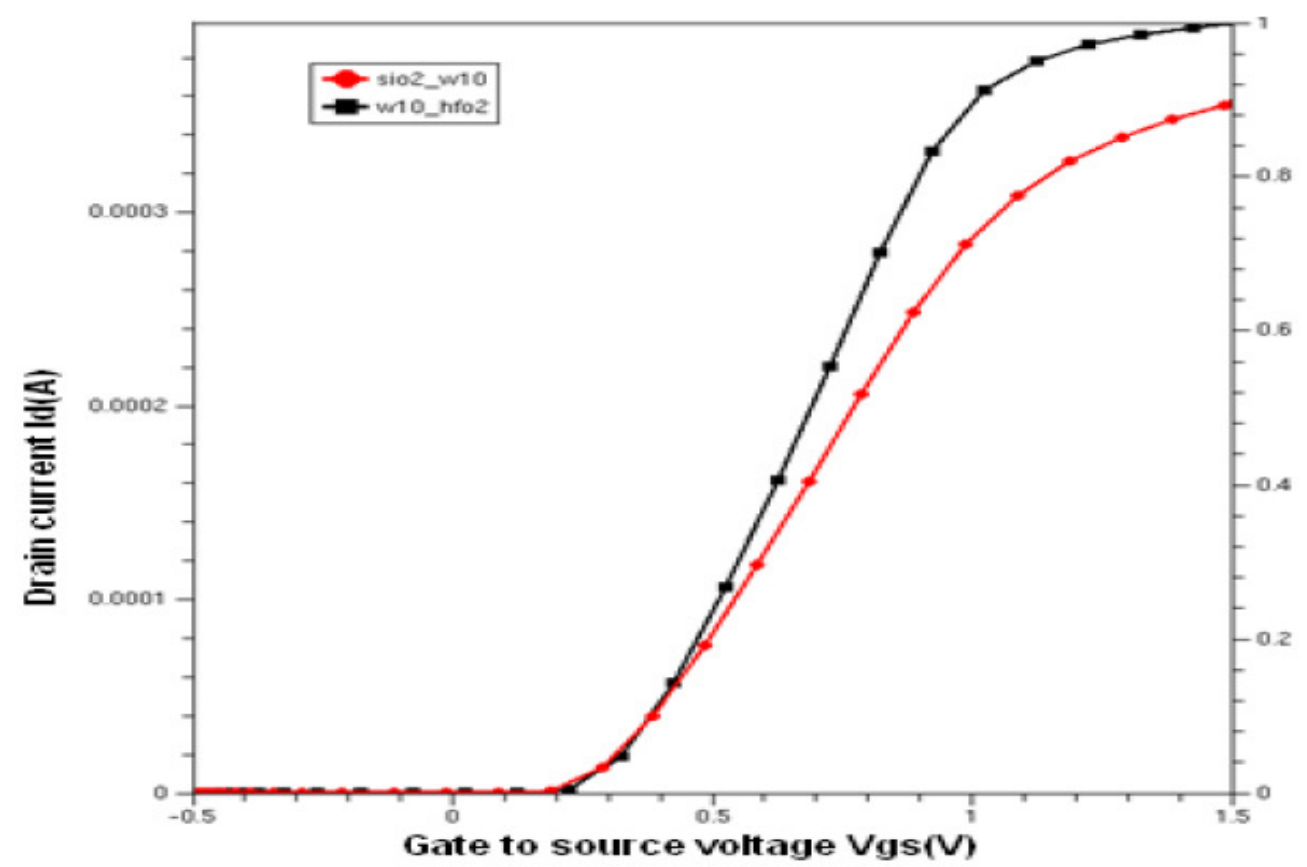

Fig. 3.8 Transfer characteristics of GAA structure with $\mathrm{SiO} 2$ and $\mathrm{HfO} 2$ gate oxide material for $10 \mathrm{~nm} \mathrm{~W}_{\text {fin }}$

The off state current is minimum in case of $\mathrm{HfO} 2$ with $\mathrm{W}_{\text {fin }}$ equal to $10 \mathrm{~nm}$ but it has low value of on state current. The on state can be increased by increase in $\mathrm{W}_{\text {fin }}(20 \mathrm{~nm})$ as the Fig. 3.9 indicate compared to the GAA structure of same size with $\mathrm{SiO} 2$ oxide material. 
International Journal of VLSI design \& Communication Systems (VLSICS) Vol.3, No.5, October 2012

Fig. 3.10, 3.11 shows the comparison between subthreshold characteristics of GAA structure with $\mathrm{SiO} 2$ and $\mathrm{HfO} 2$ as dielectric material for gate oxide and also gives the comparison among GAA structures with different value of $\mathrm{W}_{\text {fin }}$. Both the graph indicate that GAA structure with $\mathrm{HfO} 2$ oxide material gives improved Ion/Ioff ratio with the improvement in subthreshold performance.

Here, the Fin shape is cubical in GAA structure but the similar behavior can be obtained with cylindrical Fin[10]. The future device scaling can be done with these GAA structures without compromise in their performances.

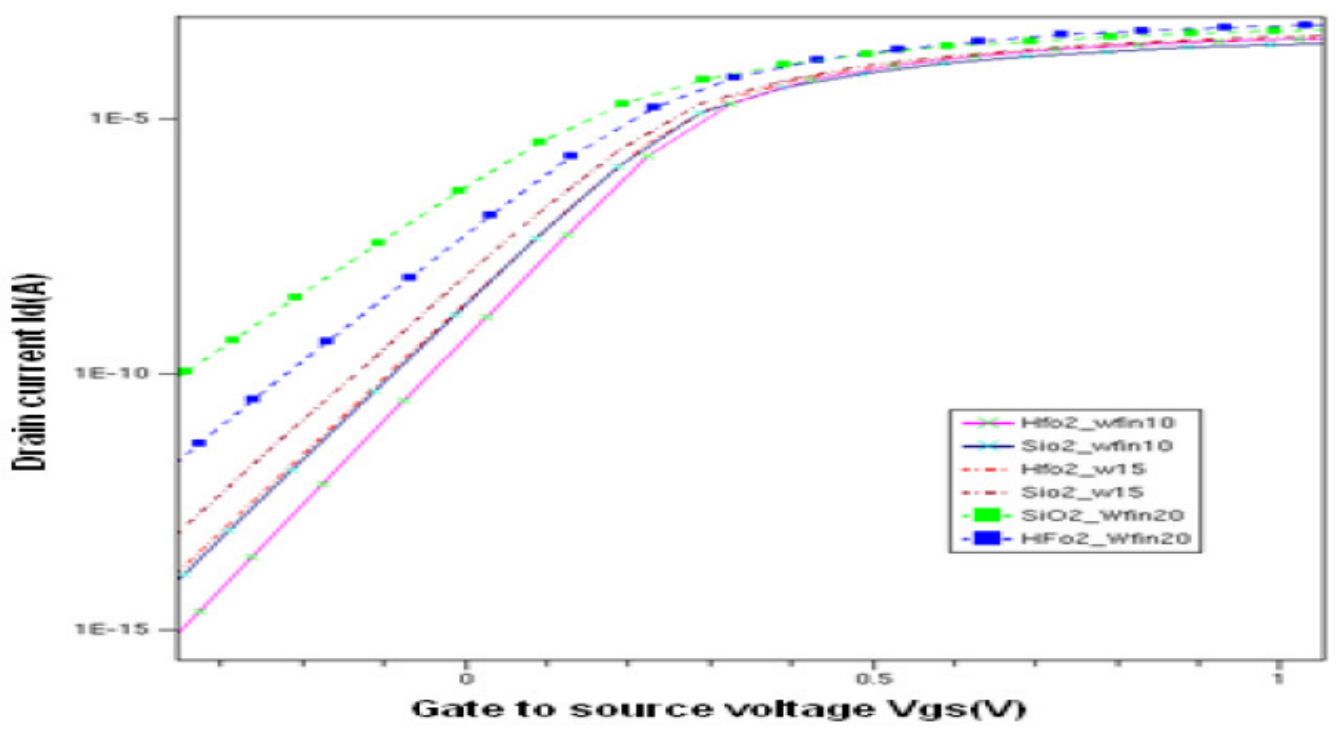

Fig. 3.9 Subthreshold characteristics of GAA with $\mathrm{SiO} 2$ and $\mathrm{HfO} 2$ gate oxide for different $\mathrm{W}_{\text {fin }}$

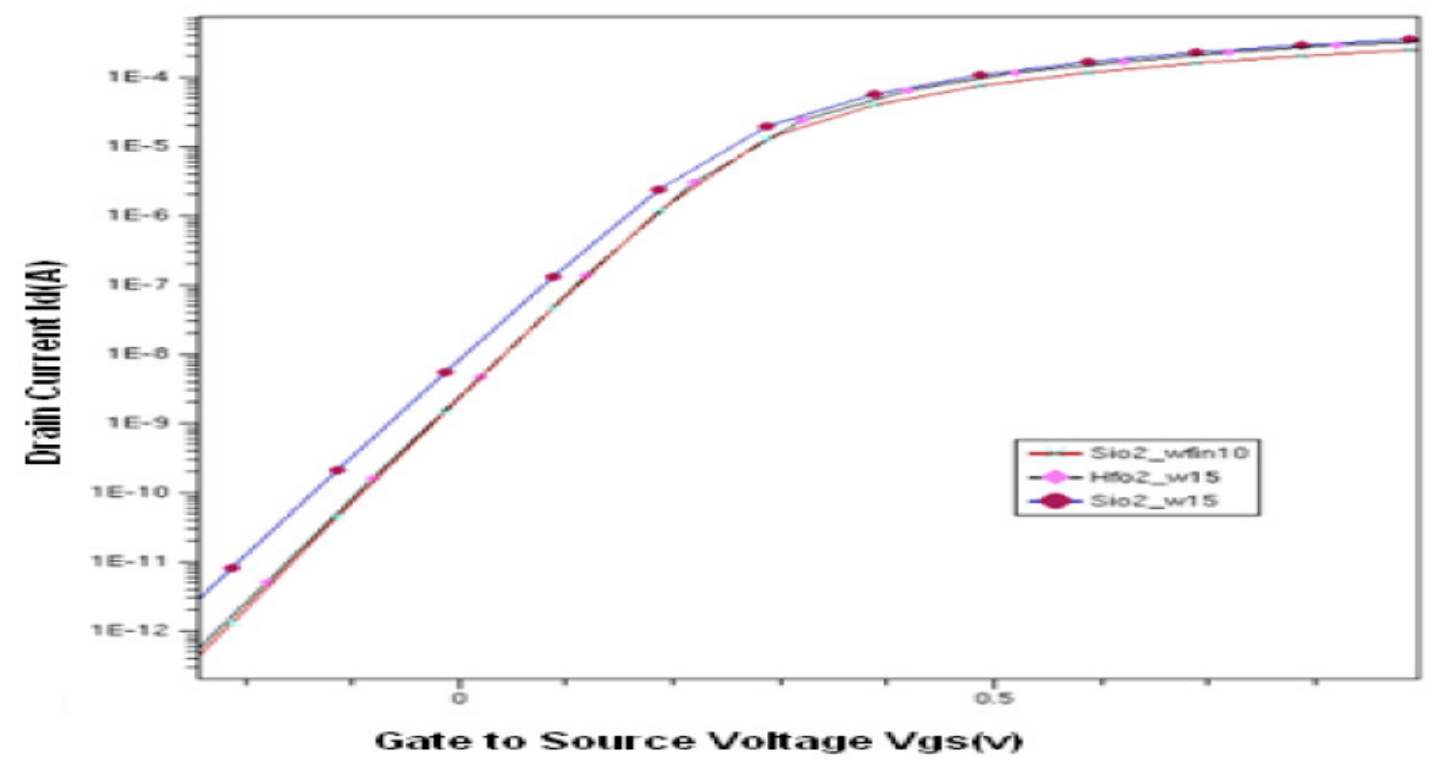

Fig. 3.10 Subthreshold characteristics of GAA with $\mathrm{SiO} 2$ and $\mathrm{HfO} 2$ gate oxide for $15 \mathrm{~nm} \mathrm{~W}_{\text {fin }}$ 
In in future GAA structures will give better replacement of $p-n$ junction diode as driving device in phase-change memory cells (PCM) with more scalability[16].

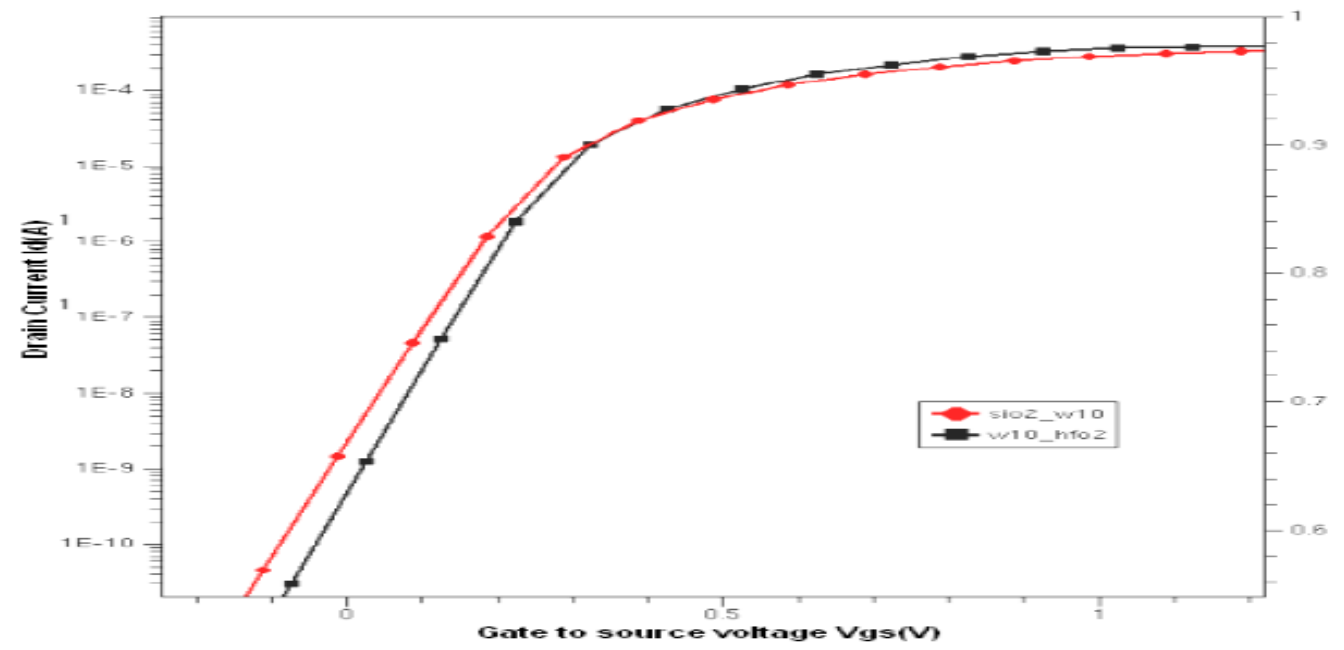

Fig. 3.11 Subthreshold characteristics of GAA with $\mathrm{SiO} 2$ and $\mathrm{HfO} 2$ gate oxide for $10 \mathrm{~nm}_{\text {fin }}$

\section{CONCLUSION}

"If we required high Fin width MOSFET, FinFET is not a good option" but if we use GAA instead of FinFET more volume inversion is obtain and after a certain Fin height the performance of GAA increases while decreasing $\mathrm{h}_{\text {fin. }}$. This paper shows that for low value of $\mathrm{h}_{\text {fin }}$, high performance GAA can be structured even for High Fin width. Therefore, the condition of low Fin width is no longer be a problem for GAA structure because of it' $s$ superior performance with high Fin width. Further we extended our results to high-K Gate oxide materials to obtain more improvement in device performance. The graph of Fig.3.9,3.10\&3.11 are showing that GAA with high K gate oxide materials has superior performance for the same Fin width. The GAA structure on Bulk can also be designed and its performance can be improved with high Fin width using high $\mathrm{K}$ dielectric gate oxide materials.

\section{REFERENCES}

[1] Jean-Pierre Colinge, "Multiple-gate SOI MOSFETs" Solid-State Electronics 48 (2004) 897-905

[2] A. Breed and K.P. Roenker, "Dual-gate (FinFET) and Tri-Gate MOSFETs: Simulation and design" ISDRS-2003, pp. 150-151, December 2003

[3] Y.-K. Choi, N. Lindert, P. Xuan, S. Tang, D. Ha, E. Anderson, T.-J. King, J. Bokor, and C. Hu, "Sub20nm CMOS FinFET technologies," in Tech. Dig. IEDM, 2001, pp. 421-424.

[4] E. J. Nowak, I. Aller, T. Ludwig, K. Kim, R. V. Joshi, C.-T. Chung, K. Bernstein, and R. Puri, "Turning silicon on its edge," IEEE Circuits Devices Mag., vol. 20, no. 1, pp. 20-31, Jan./Feb. 2004.

[5] Vishwas Jaju, Dr. Vikram Dalal "Silicon-on-Insulator Technology" EE -530, Advances in MOSFETs, Spring 2004

[6] B. S. Doyle, S. Datta, M. Doczy, S. Hareland, B. Jin, J. Kavalieros, T. Linton, A. Murthy, R. Rios, and R. Chau, "High performance fully-depleted tri-gate CMOS transistors," IEEE Electron Device Lett., vol. 24, no.4, pp. 263-265, Apr. 2003. 
International Journal of VLSI design \& Communication Systems (VLSICS) Vol.3, No.5, October 2012

[7] K. Suzuki, T. Tanaka, Y. Tosaka, H. Horie, and Y. Arimoto, "Scaling theory for double-gate SOI MOSFET"s," IEEE Trans. Electron Devices, vol. 40, no. 12, pp. 2326-2329, Dec. 1993.

[8] Gaurav Saini1, Ashwani K RanaInternational, " Physical Scaling Limits of FinFET Structure: A Simulation Study" Journal of VLSI design \& Communication Systems (VLSICS) Vol.2, No.1, March 2011

[9] J. Colinge, M. Gao, A. Romano-Rodriguez, H. Maes, and C. Claeys, "Silicon-on-insulator gate-allaround device'," in Tech. Dig. IEDM, 1990, pp. 595-598

[10] Jae Young Song, Woo Young Choi, Ju Hee Park, Jong Duk Lee and Byung-Gook Park, "Design Optimization of Gate-All-Around (GAA) MOSFETs" IEEE Transactions on Nanotechnology, VOL. 5, NO.3, MAY 2006

[11] Sentaurus Device User Guide, Synopsys, Inc., Mountain View, CA,Version Z-2007.03, Mar. 2007.

[12] International Technology Roadmap for Semiconductors (ITRS), 2007 Edition. www.itrs.net

[13] Deepesh Ranka, Ashwani K Rana, Rakesh Kumar, Yadav and Devendra Giri. The "Performance Analysis of FD-SOI MOSFET with Different Gate Spacer Dielectrics". IJCA (0975 - 8887)Volume 18-No.5, March 2011

[14] M. J. Kumar, S. K. Gupta and V. Venkatraman " compact modeling of the effects of parasitic internal fringe capacitance on the threshold voltage of high $\mathrm{k}$ dielectric nanoscale SoI MOSFETs. IEEE transaction on Electronic Devices, Vol. 52, no.4 pp. 706-711, April 2004

[15] Hui Zhao, Yee-Chia Yeo, Subhash C. Rustagi, and Ganesh Shankar Samudra . "Analysis of the Effects of Fringing Electric Field on FinFET Device Performance and Structural Optimization Using 3-D Simulation" IEEE transactions on Electronics Devices, vol. 55, no. 5, May 2008

[16] Lin Li, Kailiang Lu, Bipin Rajendran, Thomas D. Happ, Hsiang-Lan Lung, Chung Lam, and Mansun Chan, " Driving Device Comparison for Phase-Change Memory", IEEE transactions on electron devices, vol. 58, no. 3, march 2011

\section{AUTHORS}

S.L.Tripathi: She has received her B.Tech degree from Purvanchal University, Jaunpur and M.Tech from UP technical university, Lucknow . Currently working towards Phd in low power VLSI design using Multigate MOSFET structures from MNNIT, Allahabad.

Ramanuj Mishra: He has received the B.E. Degree in Electronics and Communication from RGPV in 2009 Bhopal, India. And completed M.Tech in microelectronics and VLSI design from MNNNIT Allahabad, His current research interests include of Short channel devices such as FinFETs.

Dr. R. A. Mishra is presently working as Associate Professor in the Department of Electronics and Communication Engineering, M.N.N.I.T Allahabad (U.P) India. He has 20 years teaching experience and published many papers in International Journal and Conference proceeding. His research area includes VLSI circuit, semiconductor devices and modeling and residue number system based circuit design.
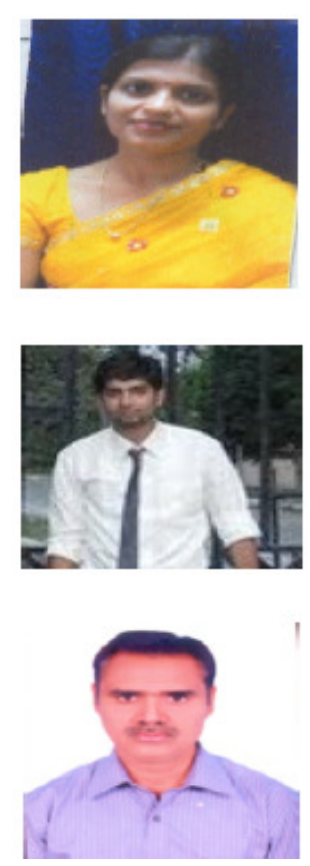